\title{
BMJ Open Effectiveness-implementation hybrid type 2 trial evaluating two psychoeducational programmes for severe hypoglycaemia in type 1 diabetes: implementation study protocol
}

\author{
Tayana Soukup, ${ }^{1}$ Louise Hull, ${ }^{1}$ Emma Lauretta Smith, ${ }^{2,3}$ Andy Healey, ${ }^{1}$ \\ Ioannis Bakolis, ${ }^{1,4}$ Stephanie A Amiel (10) , ${ }^{2,3}$ Nick Sevdalis, ${ }^{1}$ PWD Group ${ }^{5}$
}

To cite: Soukup T, Hull L, Smith EL, et al. Effectivenessimplementation hybrid type 2 trial evaluating two psychoeducational programmes for severe hypoglycaemia in type 1 diabetes: implementation study protocol. BMJ Open 2019:9:e030370. doi:10.1136/ bmjopen-2019-030370

- Prepublication history for this paper is available online. To view these files, please visit the journal online (http://dx.doi. org/10.1136/bmjopen-2019030370).

Received 19 March 2019 Revised 13 August 2019 Accepted 02 September 2019

Check for updates

(C) Author(s) (or their employer(s)) 2019. Re-use permitted under CC BY-NC. No commercial re-use. See rights and permissions. Published by BMJ.

For numbered affiliations see end of article.

Correspondence to

Dr Tayana Soukup;

tayana.soukup@kcl.ac.uk

\section{ABSTRACT}

Introduction Two of the most acute and feared complications in type 1 diabetes (T1D) are hypoglycaemia and severe hypoglycaemia (SH). While impaired awareness of hypoglycaemia (IAH) can lead to SH with cognitive and motivational barriers implicated, the available education does not integrate behavioural change techniques to address these. A novel Hypoglycaemia Awareness Restoration Programme despite optimised care (HARPdoc) is currently being tested against an established blood glucose awareness training (BGAT) within a parallel, two-arm, group randomised, blinded trial (with its own protocol; NCT02940873) with adults with T1D whose problems with hypoglycaemia and SH have persisted despite otherwise optimised insulin management. While both programmes are aimed at reducing hypoglycaemia, $\mathrm{SH}$ and IAH, it is the former that integrates behavioural change techniques.

The aim of the current (implementation) study is to evaluate delivery of both HARPdoc and BGAT and explore associations between implementation outcomes and trial endpoints; as well as to develop an evidence-based implementation blueprint to guide implementation, sustainment and scale-up of the effective programmes.

Methods and analysis Guided by the implementation science tools, frameworks, methods and principles, the current study was designed through a series of focus groups $(n=11)$ with the key intervention stakeholders $(\mathrm{n}=28)$-including (1) individuals with lived experience of T1D, IAH and a pilot version of the HARPdoc $(n=6)$ and (2) diabetes healthcare professionals $(n=22)$. A mixed-methods approach will be used throughout. Stakeholder engagement has underpinned study design and materials to maximise relevance, feasibility and impact.

Ethics and dissemination The protocol has been reviewed and received ethical approval by the Harrow Research Ethics Committee (18/L0/1020; 240752) on 1 October 2018. The findings will be submitted to a peerreviewed journal and presented at scientific meetings. Trial registration number NCT02940873; Pre-results.
Strengths and limitations of this study

- Study design driven by the intervention stakeholders including people with lived experience and the healthcare professionals.

- Study design driven by implementation science theories, models and principles.

- Mixed-method approach.

- Assessments will not be conducted prior to intervention implementation.

\section{INTRODUCTION}

\section{Type 1 diabetes and hypoglycaemia}

Type 1 diabetes (T1D) is a condition of deficiency of endogenous insulin secretion characterised by hyperglycaemia (blood glucose above normal), requiring administration of exogenous insulin for regulation of blood glucose levels. ${ }^{1}$ However, one of the most acute and feared complications of the insulin therapy in T1D is hypoglycaemia (blood glucose below normal), and in particular, severe hypoglycaemia $(\mathrm{SH})$, whereby blood glucose is so low that an individual may lose consciousness, or become acutely cognitively impaired to the degree that they are unable to take appropriate glucose treatment to raise their blood glucose and so require someone else to give treatment to them. ${ }^{23}$ Each year, $22 \%-46 \%$ of people with T1D experience $\mathrm{SH}^{45}$ with common presentations including confusion, coma and seizure. ${ }^{6-8} \mathrm{SH}$ can lead to mortality: $4 \%-10 \%$ of adults with T1D under the age of 40 die as a result of $\mathrm{SH}^{9-11}$ In England and Wales alone, this equates to $500-1391$ deaths annually, ${ }^{12} 13$ as well as 14387 hospital admissions, 660 day cases and 65601 bed days (between 2012 and 2013). ${ }^{14}$

A significant factor affecting the successful management of hypoglycaemia in adults with 
T1D is impaired awareness of hypoglycaemia (IAH), that is, reduction in intensity or delayed onset of the physiological symptoms (eg, dizziness, sweating, palpitations) that serve a vital function of prompting an individual to ingest glucose to raise blood glucose to normal limits. Not surprisingly, thus, IAH is a significant risk factor for $\mathrm{SH}$, and linked to a six to eightfold increase in the frequency of SH events. ${ }^{3}$ Evidence shows that $25 \%-40 \%$ of adults with T1D have IAH. ${ }^{1516}$

Cognitive and motivational factors have been found to be associated with IAH in a subset of individuals who express low concern for hypoglycaemia and $\mathrm{SH}$, yet they are at high risk of such events. ${ }^{17}$ Certain cognitions or beliefs, such as normalising the presence of IAH or underestimating the consequences of hypoglycaemia, excessive concern about the possible consequences of hyperglycaemia, and wanting to avoid the sick role have been identified as barriers to avoiding hypoglycaemia and regaining awareness of associated symptoms. ${ }^{17-19}$ The presence of such beliefs is thought to explain why one-third of people with T1D continue to experience $\mathrm{SH}$ and IAH, despite receiving optimal education in insulin self-management. ${ }^{16}$

Several education programmes,${ }^{20}$ such as the German Diabetes Teaching and Training Programme, ${ }^{21}$ and the UK's Beta Cell Education Resources for Training ${ }^{22}$ and Dose Adjustment for Normal Eating (DAFNE), ${ }^{23}$ aim to provide a foundation of knowledge and skills in flexible and safe insulin management, and in the UK, these are offered to all adults with T1D. While these programmes do not explicitly focus on IAH or $\mathrm{SH}$, they provide more general training in diabetes self-management and include information for the general treatment and prevention of hypoglycaemia. ${ }^{20}$ However, improvement in glycaemic outcomes following completion of such programmes is unclear. For instance, individuals who have completed DAFNE $^{23}$ do not always experience improved or sustained glycaemic outcomes, indicating that a more targeted approach may be required for individuals who continue to experience problematic hypoglycaemia despite education on the general principles. ${ }^{24}$

Structured education with specific focus on hypoglycaemia management and avoidance has, therefore, been developed, such as, blood glucose awareness training $(\text { BGAT })^{25-30}$ and self-management-oriented education programme, ${ }^{31}$ which seem to have similar benefit on hypoglycaemia risk as the more generic programmes, where these have been specifically compared. ${ }^{20}$ Another programme is DAFNE-Hypoglycaemia Awareness Restoration Training (DAFNE-HART), ${ }^{32}$ which was specifically designed to address the ongoing needs of people with T1D who continued to experience $\mathrm{SH}$ and IAH after completing DAFNE. ${ }^{23}$

DAFNE-HART was based on the research identifying abnormal activation of cortical brain regions in response to hypoglycaemia in people with IAH and the research identifying low concern, and unhelpful cognitions about hypoglycaemia among people with IAH. ${ }^{33}$ It sought to address cognitive and motivational barriers associated with IAH and SH, especially in adults with T1D who continue to experience $\mathrm{SH}$ despite optimal previous insulin regimens and educational programmes. ${ }^{33}$ However, to further enhance diabetes education, it has been argued that future programmes should incorporate behavioural change and other psychologically informed techniques to address cognitive and motivational barriers associated with IAH and SH. ${ }^{17} 192023$

To address this goal, a novel education programme has been further developed from DAFNE-HART, namely, Hypoglycaemia Awareness Restoration Programme despite optimised care (HARPdoc), which is currently being tested against the BGAT programme within a parallel, two-arm, group randomised, blinded clinical trial. ${ }^{33}$ Both HARPdoc and BGAT are group therapies aimed at reducing hypoglycaemia, $\mathrm{SH}$ and IAH in adults with T1D who are continuing to experience SH and are being tested in the trial only in people whose problems with hypoglycaemia have persisted despite otherwise optimised management of their insulin treatment regimens and educational programmes. For more detail on the two education programmes tested with the trial and evaluated as part of the implementation, please see the Methods section.

\section{Aim and objectives}

The aim of the study is to assess the way in which HARPdoc and BGAT are delivered in the context of the clinical trial sites, facilitating understanding of the potential link between the delivery of the programmes under investigation and the expected improvements in $\mathrm{SH}$ rates. This will be achieved in a qualitative, but also quantitative manner which will allow us to explore the relationship between the implementation of the programmes and the clinical trial's outcomes. Ultimately, this will enable the development of an implementation blueprint to facilitate future implementation after the trial is completed.

The study will address the following research questions for both education programmes from the perspectives of the intervention stakeholders (including adults with T1D with problematic hypoglycaemia, their relatives, as well as diabetes physicians, educators, psychologists and support staff):

1. To what extent are the programmes acceptable, appropriate and feasible?

2. To what extent were the programmes delivered as intended (fidelity of delivery)?

3. To what extent were the programmes received as intended (fidelity of receipt)?

4. How willing are key stakeholders to adopt the programmes, and what are the anticipated facilitators and barriers to adoption?

5. What are the anticipated facilitators and barriers to sustainment of the programmes long term (ie, after the trial is completed)?

6. What are the costs associated with implementing the programmes? 
7. What are unintended consequences (positive/negative) associated with programmes?

8. What are the contextual enablers and barriers associated with the implementation of the programmes?

9. What implementation strategies were used within individual sites to improve implementation of the programmes?

10. Is there a relationship between implementation and effectiveness outcomes?

\section{METHODS}

\section{Design}

The design of our study is an effectiveness-implementation hybrid type $2,{ }^{35}$ placing equal focus on investigating the effectiveness of the two education programmes as part of the HARPdoc trial, ${ }^{33} 34$ while simultaneously investigating the implementation of the programmes as part of the current protocol. We plan to apply a mixed-methods approach incorporating a variety of qualitative and quantitative data collection techniques, including surveys, structured interviews and psychometrically established measurement scales.

The study design was (1) shaped by implementation science concepts and frameworks, (2) refined and informed by the intervention stakeholders and (3) guided by a tool specifically developed to guide the design of implementation research. In what follows, we describe the design process in more detail.

First, the design was shaped by a number of complementary implementation science frameworks that have been selected in light of the aims and objective of the study:

- Medical Research Council framework for evaluating complex interventions ${ }^{36-39}$ to guide and inform the study design and processes (questions 1-10).

- Reach Effectiveness Adoption Implementation Maintenance (RE-AIM) framework $^{40}$ to guide selection of implementation outcomes in conjunction with Proctor et at ${ }^{41}$ definitions of these outcomes (questions 1-7).

- Consolidated Framework for Implementation Research (CFIR $)^{42}{ }^{43}$ to develop semistructured interview topic guides (see Methods section) and subsequently guide the coding and analysis of barriers and facilitators to programme implementation (question $8)$.

- Implementation strategies compendium reported by Powell et $a l^{44}$ (question 9).

Second, the design was refined by the key intervention stakeholders, including (1) individuals with lived experience of T1D, hypoglycaemia unawareness and an earlier version of the HARPdoc, namely, DAFNE-HART (this is the patient and public involvement group for the study or people with diabetes (PWD) group) and (2) healthcare professionals (HCPs) involved in the delivery of the interventions under investigation. The stakeholders critically reviewed for relevance, feasibility and clarity a selection

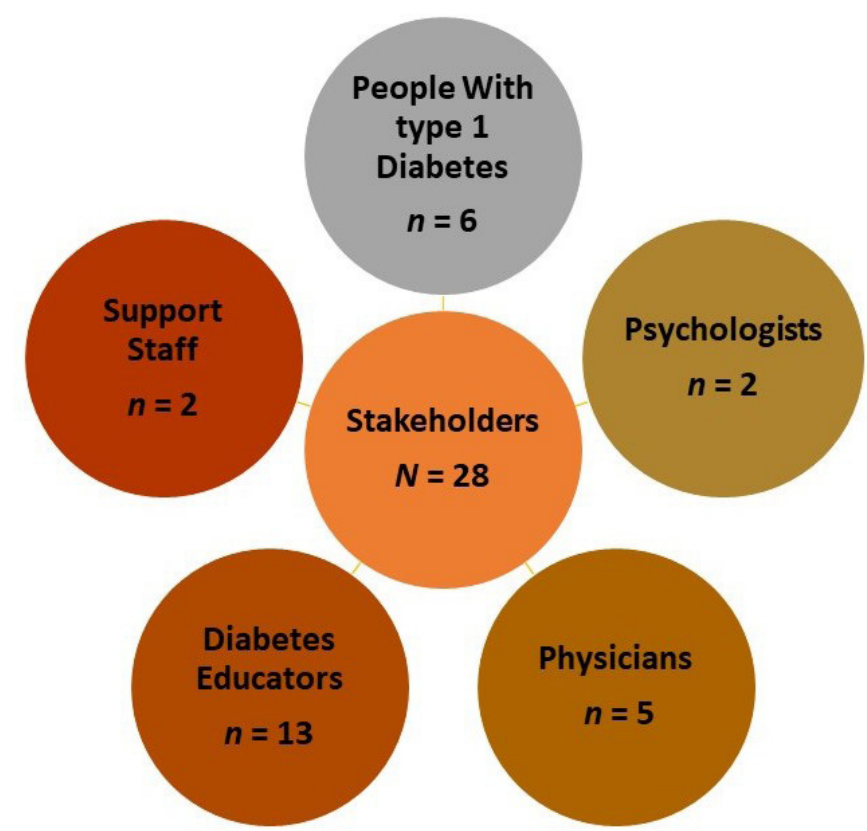

Figure 1 Stakeholder groups that informed the design of the study.

of (1) factors commonly assessed and reported in implementation science research ${ }^{40-44}$ and (2) qualitative and quantitative assessment tools and methods (eg, surveys and interview topic guides described in more detail in the data collection plan section), and their measurement time points. Figure 1 shows stakeholder groups involved in the research design process.

Specifically, the outcome measures and materials were presented and reviewed, within an iterative process of continuous development and refinement, by the key stakeholders $(n=28)$, including the people with lived experience of T1D, IAH and education courses $(n=6)$, as well as the HCPs $(n=22)$ across participating diabetes centres $(\mathrm{UK}=4 ; \mathrm{USA}=1)$ in a series of focus groups $(\mathrm{n}=11)$ between October and December 2017. Two 1.5-hour long sessions were conducted with same group of representatives of people with T1D, and nine 1-hour long sessions with different HCPs. Following each meeting, stakeholders' feedback was incorporated, and the final versions of the study materials further codesigned and refined. The final selection of the stakeholder-driven outcome measures, materials and methods (eg, surveys and interview topic guides) proposed in this protocol was thus a result of an iterative development process where feedback from stakeholders fed directly into the development and refinement of the study design.

Lastly, the Implementation Science Research Development (ImpRes) tool and guide ${ }^{45} 46$ was used to guide the study design and the stakeholder-driven planning process. ImpRes is a newly developed instrument that helps researchers to design high-quality implementation research, based on best evidence and expert recommendations. ImpRes is theory agnostic in that it does not advocate or require use of a specific implementation 
framework; rather, ImpRes guides research teams through the various elements that a well-designed implementations study should consider, based on current literature and expert inputs. These include: choice of appropriate frameworks, articulation of patient, service and implementation outcomes and how they are to be assessed, articulation of the stakeholders of a study, a proforma for the definition of implementation costs and other elements. Using ImpRes, we were able to:

- Establish a set of most relevant and feasible implementation outcome measures (drawing on implementation outcomes taxonomy, ${ }^{41}$ implementation strategies ${ }^{44}$ and RE-AIM framework. ${ }^{40}$

- Identify the most relevant and feasible validated surveys for the study.

- Formulate instructions to participants for the selected validated pragmatic surveys.

- Develop a set of interview topic guides drawing on implementation science frameworks, including the implementation outcomes taxonomy, ${ }^{41}$ implementation strategies taxonomy, ${ }^{44}$ RE-AIM framework ${ }^{40}$ and CFIR. ${ }^{42} 43$

- Identify appropriate and most relevant participant groups for the study.

- Develop stakeholder-centred participant information sheets and consent forms.

Using the ImpRes tool and guide ${ }^{45} 46$ ensured that the study design was (1) informed by implementation science frameworks, concepts and measures, and (2) informed by key stakeholders (figure 1). This process was conducted within the context of a highly intricate study design of a parallel, two-arm, group randomised, blinded clinical trial where pragmatic considerations for the current study had to be made (eg, timelines for evaluating implementation outcomes).

\section{Setting}

This is an international, multisite study based in the UK and USA. In the UK, participants will be recruited from four diabetes centres, across four National Health Service Trusts in England-two in the greater London area, one in Dorset and one in South Yorkshire, while in the USA from a single diabetes centre in Massachusetts between July 2018 and December 2019. The participating sites are specialist care diabetes centres that (1) provide structured education for T1D and therefore have on-site diabetes educators, (2) contain the necessary clinical capability and expertise in the hypoglycaemia management and (3) routinely receive tertiary referrals for problematic hypoglycaemia and therefore act as a channel for recruiting this very niche group of adults with $\mathrm{T} 1 \mathrm{D}$ and problematic hypoglycaemia.

\section{Participants}

Stakeholders of the HARPdoc trial ${ }^{33} 34$ and the two educational programmes under investigation (see Trialled educational programmes) form the participant group of the current study. They include course participants who are people with T1D with problematic hypoglycaemia, relatives of people enrolled in the trial (HARPdoc arm only since it encompasses relative session in week 6), as well as the diabetes HCPs. People with T1D include participants who were found eligible for the trial and have either (1) fully or (2) partially attended either one of the two programmes or (3) have declined and not attended either one of the programmes. The HCPs comprise diabetes educators, physicians, psychologists and support staff. For the quantitative part of data collection, a census approach will be used, and the entire available population will be approached, that is, all patients $(\mathrm{n}=96)$ participating in the trialled interventions and all HCPs $(n=28)$ involved in the delivery of these interventions. For the qualitative part, availability sampling will be used within the subgroups of the recruited population according to the site and course, that is, those HCPs and patients within the participating hospitals recruited into or delivering one of the two programmes who are available and willing to partake in the interview. The latter will culminate in sample of approximately 32 patients in total ( 4 sites $\mathrm{x} 2$ patients $\mathrm{x} 2$ types of courses $\mathrm{x} 2$ sets of courses per site) and 28 HCPs. A convenience sampling will be used to recruit people who declined to take part in the programme.

\section{Patient and public involvement}

Patient and public involvement group, that is, PWD group for the study (as described under the Design section and in figure 1) was involved in the development of the study presented in this protocol from the very conception through to the selection of outcomes, design of measures including topic guides, and formulating documents for ethical approval (including information sheets and consent forms), as well as this protocol itself. A detailed description of how the PWD group for the study has shaped the design of this study is outlined under the Design section and in figure 1 . The group will also be actively involved in the analysis, write-up and subsequent dissemination of the study findings, going forward.

\section{Trialled educational programmes}

The clinical trial testing the effectiveness of the programmes is currently ongoing and blinded, ${ }^{33} 34$ and therefore, in what follows, we provide only a high-level overview of the programmes (see table 1) with detailed information on the curricula purposefully omitted to prevent contamination.

The trial is testing two group programmesHARPdoc $^{34}$ and BGAT. ${ }^{25-30}$ The former incorporates structured education relevant to hypoglycaemia management, avoidance and strategies to restore hypoglycaemia awareness with behavioural change and psychologically informed techniques aimed to address the cognitive barriers to hypoglycaemia avoidance associated with IAH. It also involves family members in the final session. The curriculum is delivered over 6 weeks in a combination of group and individual 
Table 1 Differences and similarities between HARPdoc and BGAT structure and delivery within the trial

\begin{tabular}{ll}
\hline Structure and delivery & HARPdoc \\
\hline Programme duration & 6 weeks \\
Programme structure & 4 weekly full-day group sessions, weeks 1, 2, 3 \\
& and 6 \\
& 1 weekly one-to-one telephone session in weeks \\
& 4 and 5
\end{tabular}

\begin{tabular}{|c|c|c|}
\hline Group size & $5-10$ & $5-10$ \\
\hline Diabetes centres & 4 & 4 \\
\hline $\begin{array}{l}\text { No of courses per diabetes } \\
\text { centre }\end{array}$ & $2-4$ & $2-4$ \\
\hline $\begin{array}{l}\text { Sample size } \\
\text { (overall } 96 \text { course participants) }\end{array}$ & $\begin{array}{l}\text { Total of } 48 \text { course participants } \\
4-8 \text { participants per course }\end{array}$ & $\begin{array}{l}\text { Total of } 48 \text { course participants } \\
4-8 \text { participants per course }\end{array}$ \\
\hline Educator training & $\begin{array}{l}\text { 3-day workshop ( } 1 \text { day to standardise training } \\
\text { in hypoglycaemia prevention, and } 2 \text { days to } \\
\text { train in behavioural change and psychologically } \\
\text { informed strategies) } \\
\text { 1-day refresher course after first courses; } \\
\text { scheduled supervision by clinical psychologist }\end{array}$ & $\begin{array}{l}\text { 3-day workshop ( } 1 \text { day to standardise } \\
\text { training in hypoglycaemia prevention, } \\
\text { and } 2 \text { days to review and update the } \\
\text { curriculum with the clinical psychologist } \\
1 \text { day refresher course after first courses; } \\
\text { supervision optional (on request) }\end{array}$ \\
\hline Educators per course & 2 educators per course & 1 educator per course \\
\hline Follow-ups & $\begin{array}{l}\text { 2-hour group sessions delivered at 3, } 6 \text { and } 12 \\
\text { months after the course }\end{array}$ & None \\
\hline Programme adherence & $\begin{array}{l}\text { First } 3 \text { group sessions, and } 1 \text { one-to-one } \\
\text { session }\end{array}$ & First 3 group sessions \\
\hline
\end{tabular}

BGAT, Blood Glucose Awareness Training (comparator); HARPdoc, Hypoglycaemia Awareness Restoration Programme despite optimised care.

sessions facilitated by two experienced diabetes educators, trained to deliver the intervention and supported in its delivery by the study clinical psychologist. The programme was piloted in the DAFNE-HARP study, and amended in the light of the experience of that study. ${ }^{32}$ A significant and substantial reduction of SH from 10 to 0.5 episodes per year was document in the pilot study, as well as a significant reduction in worry and improved behavioural avoidance of hyperglycaemia. ${ }^{32}$

The latter is a UK's National Institute for Health and Care Excellence ${ }^{25}$ recommended programme focused on recognising and reducing both, hypo and hyperglycaemia and empowering people with T1D to anticipate, detect, treat and prevent extremes in blood glucose levels. ${ }^{25-30}$ The curriculum is delivered by one experienced diabetes educator, trained in its delivery by one of the clinical psychologists who originally designed it. For the purposes of the trial, the BGAT timetable has been modified to be delivered in the same time frame as the HARPdoc, with the original eight 2-hour sessions delivered over 6 weeks, and as two sessions in 1 day in weeks 1, 2, 3 and 6 . While BGAT has proved successful in improving glycaemic control, hypoglycaemia

\section{BGAT}

6 weeks

$8 \times 2$ hours group sessions delivered as 2 sessions delivered in 1 day in weeks 1, 2, 3 and 6

1 optional one-to-one telephone session in either week 4 or 5 $5-10$

Total of 48 course participants

(1 day to standardise curriculum with the clinical psychologist 1 educator per course

and 6

1 optional one-to-one telephone session in week 4 or 5

First 3 group sessions 
Table 2 Data collection plan for the implementation study: assessment objectives, data, instruments, timeline and participants

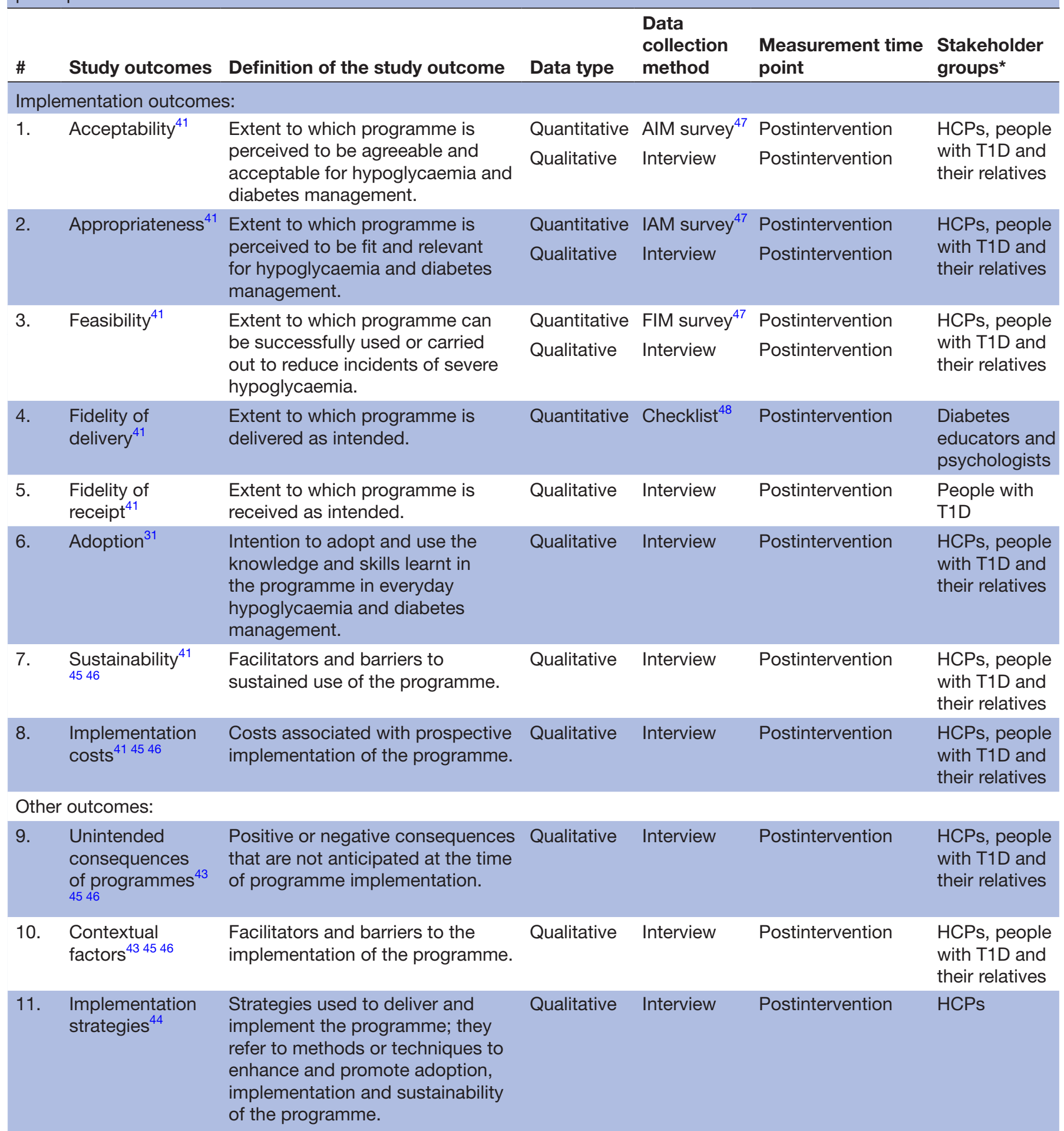

${ }^{*} \mathrm{HCPs}$, healthcare professionals including diabetes educator, physician, psychologist and administrative support.

AIM, Acceptability of Intervention Measure; FIM, Feasibility of Intervention Measure ${ }^{47}$; IAM, Intervention Appropriateness Measure; T1D, type 1 diabetes.

by key stakeholders, that is, people with $\mathrm{T} 1 \mathrm{D}$, relatives of people enrolled on HARPdoc and diabetes HCPs who will assess the degree to which they perceive the programmes acceptable, feasible and appropriate for diabetes and hypoglycaemia management. In addition, one-to-one semistructured interviews with stakeholders will also be conducted. The interviews will complement the survey data and explore in more detail stakeholders' perceptions and experiences of HARPdoc and BGAT in terms of acceptability, appropriateness and 
feasibility. Both surveys and interviews will occur at one time point, after the programme completion.

Question 2: to what extent were programmes delivered as intended by diabetes educators (fidelity of delivery)?

Relevant sections of audiotapes from both the BGAT and HARPdoc group sessions across each of the sites will be assessed; this will form the fidelity of delivery part of the study that will be led by the clinical study team. An observational assessment tool has been adapted and refined by the clinical study team from the AMIGOS. ${ }^{48}$ Two trained and experienced independent raters will use the tool to assess the delivery of the programmes by the diabetes educators from the audio recordings of individual sessions across each site.

Question 3: to what extent were programmes received as intended by adults with $\mathrm{T1D}$ (fidelity of receipt)?

Literature acknowledges patients as active participants in health interventions with the fidelity of receipt focused on the way the intervention is received by the individuals as highly important although seldom addressed in health research. ${ }^{49}$ We will, therefore, explore the fidelity of receipt, that is, fidelity with which the content of HARPdoc and BGAT is received by the adults with T1D, as part of the one-to-one semistructured interview (which will also explore acceptability, appropriateness and feasibility of the programmes as described in the section Trialled educational programmes). The participants who have had a useful exposure to the programmes and have either fully or partly (at least the first 3 days attended) completed one of the programmes will be recruited. We will explore their views and experience of the programme in relation to the extent they feel able to:

- Engage with the programme content and their group.

- Understand the content delivered to them by the diabetes educators.

- Acquire and apply the programme skills to their everyday management of hypoglycaemia.

This will help advance our knowledge of the programmes in terms of how easy or difficult it is for individuals to engage with the content, as well as to understand and apply the skills acquired as part of HARPdoc and BGAT to manage hypoglycaemia.

\section{Question 4: how willing are key stakeholders to adopt the} programmes?

As part of the interviews with the key stakeholders (described above in detail), we will explore the intention to adopt HARPdoc and BGAT from two very specific standpoints. First, from the perspective of the provider or diabetes HCPs (which is commonly explored in health research), ${ }^{41}$ where the interview will explore the extent to which the provider intends to adopt the programme (eg, deliver the programme again or refer people onto such programme) after the trial is completed.

Second, we will explore the intention to adopt from the perspective of adults with T1D who are at the receiving end and active participants in the intervention (far less commonly explored in research). ${ }^{41}$ We plan to explore the extent to which adults with T1D intend to (1) use the intervention skills in their everyday diabetes management and (2) use such a programme again or recommend it to other individuals with T1D and problematic hypoglycaemia.

In addition, we plan to interview participants who, although eligible, chose not to take part in HARPdoc and BGAT. This will allow us to explore reasons that may potentially prevent adults with T1D and problematic hypoglycaemia in participating in such programmes in future. This is important in terms of exploring the reach ${ }^{30}$ of both interventions (ie, the absolute number, proportion and representativeness of individuals who are willing to participate in a given initiative). ${ }^{30}$

\section{Question 5: what are the potential facilitators and barriers to} sustainment of the programmes long-term after the trial is complete and an effective programme identified?

As part of the one-to-one interview with the key stakeholders (described above in detail), we will explore potential facilitators and barriers to sustained use of HARPdoc and BGAT after the trial is completed from the perspectives of providers (ie, diabetes HCPs) as well as the course participants who are adults with T1D and the relatives of people in the HARPdoc arm of the trial who attended the last week of the programme.

With the providers, the focus will be on understanding the potential facilitators and barriers to the implementation of HARPdoc and BGAT into the local services after the trial is completed. In contrast, with the adults with T1D who have either fully or partially completed one of the programmes as well as the relatives, the focus will be on exploring the potential and experienced facilitators and barriers to sustained use of knowledge and skills gained in long-term hypoglycaemia management.

\section{Question 6: what are the costs associated with implementing the} programmes to the key stakeholders?

One-to-one interviews with the key stakeholders will also explore costs, in terms of time and money, associated with implementing HARPdoc and BGAT. With the HCPs, the focus will be on costs incurred as a result of the delivery, preparation and training in relation to the programme, while with adults with T1D and the relatives, the focus will be on the costs incurred attending and completing the programme.

\section{Question 7: are there any unintended consequences associated} with either one of the programmes?

One-to-one interviews with the key stakeholders will also explore any unintended consequences, both positive and negative, associated with HARPdoc and BGAT. Unintended consequences will be explored from the perspectives of the HCPs, adults with T1D and their relatives, in relation to how the programme has impacted on them and the people around them. 
Question 8: what are the contextual enablers and barriers associated with the implementation of the programmes?

The contextual enablers and barriers to intervention implementation that stakeholders have experienced will be explored and assessed in two ways: interviews with the key stakeholders and meeting minutes between HCPs involved in the trial.

One-to-one interviews with the key stakeholders (described above) will explore context of HARPdoc and BGAT in more detail from the perspectives of the HCPs, adults with T1D and the relatives. They will focus on exploring the factors that facilitated delivery or present a particular challenge to delivery.

Minutes from meetings taking place with the HCPs as part of the trial management processes will be assessed for contextual factors affecting delivery of interventions using qualitative content analysis. This will help us identify any potential barriers or facilitators to post-trial adoption, implementation and sustained use of the successful programme.

\section{Question 9: what implementation strategies were used within} individual sites to improve the implementation of the programmes? As part of the one-to-one interviews with the diabetes HCPs (described above), we will explore and identify implementation strategies (ie, methods or techniques used to enhance and promote adoption, implementation and sustainability of an intervention $)^{44}$ used within individual sites during the trial in order to improve delivery and implementation of the programmes. In addition, we will also explore any potential strategies that may be important to consider by the local sites wishing to implement HARPdoc and BGAT in future after the trial is completed.

\section{Question 10: what is the relationship between the implementation} and effectiveness outcomes of the trial?

Clinical outcome data contain information on the primary outcome, that is, the number of $\mathrm{SH}$ events over the preceding year, as well as at 12 and 24 months postcourse (as recorded in the anonymised $\mathrm{SH}$ recall form), and the secondary outcomes (eg, hypoglycaemia awareness score) in people with T1D taking part in the programmes. These data will be examined in relation to the pragmatic implementation outcome (acceptability, feasibility and appropriateness) survey data. This assessment will enable us to explore the relationship between the implementation and the effectiveness of the two arms of the trial.

\section{Data analysis}

Data will be analysed using quantitative and qualitative approaches.

\section{Quantitative analysis}

Descriptive statistics of survey and clinical trial data will be provided. Parametric and non-parametric tests will be employed to compare the survey responses between the two arms of the trial while controlling for a variety of demographic and socioeconomic variables, including the diabetes centres. Random intercept linear, logistic and Poisson regression models (depending on the distribution of the outcome) will be used to explore the relationship between implementation data and clinical trial outcome data. Mediation analysis with the use of structural equation models will also be employed to understand the potential pathways in which implementation has an impact on the effectiveness of the two suggested education programmes. An implementation by treatment interaction will be included to allow the effect of implantation to differ at each arm of the trial and across different diabetes centres. All analyses will be conducted in STATA V.14.1.

\section{Qualitative analysis}

Interviews with course participants and HCPs as well as trial's meeting minutes will be analysed qualitatively, using inductive and deductive content analysis approach until saturation is reached. ${ }^{50}$ Analysis will be conducted according to the intervention (BGAT and HARPdoc), and the diabetes centre, especially UK versus USA due to differences in the healthcare systems. We will use the CFIR $^{4142}$ to guide the coding and analysis (ie, framework analysis) of interview data to identify barriers and facilitators to the implementation and sustainment of HARPdoc and BGAT. This approach has been used previously, that is, CFIR has been applied postimplementation to investigate facilitators and barriers to implementation among stakeholders who had already adopted and implemented an innovation, thus identifying determinants of implementation post hoc. ${ }^{41}$ While we are aware that this approach is not entirely consistent with Damschroder's guidance stipulating that CFIR should be applied preimplementation to investigate facilitators/barriers to implementation, ${ }^{41}{ }^{42}$ given the constraints of conducting the effectiveness-implementation hybrid type 2 where equal focus is placed on both, implementation and effectiveness, it is not possible to follow this guidance. However, we strongly believe that our approach is of value in identifying actual, rather than anticipated, facilitators and barriers to the implementation and sustainment of HARPdoc and BGAT.

In line with Damschroder and Hagedorn's guidance, ${ }^{42} 43$ we plan to link determinants of implementation to implementation outcomes as part of the quantitative analyses described above where we propose to link the pragmatic implementation outcome survey data with the trial outcome data with the logistic and Poisson regression models.

\section{DISCUSSION}

This is a stakeholder-driven, mixed-methods, multisite, international project aimed at assessing the way in which two education programmes, HARPdoc and BGAT, implemented within an effectiveness-implementation hybrid type 2 trial are delivered in participating diabetes centres 
based in the UK and USA. Such evaluation will facilitate understanding of the association between programme implementation and the trial's end points. In the process, we will address a specific set of implementation objectives:

- Understand the extent to which the programmes are perceived to be acceptable, appropriate and feasible to key stakeholders.

- Understand the extent to which the programmes are delivered and received as intended.

- Identify contextual enablers and facilitators to the implementation of the programmes.

- Identify potential barriers and facilitators to sustainment and intention to adopt the programmes longterm after the trial is completed.

- Identify any unintended consequences and understand implementation costs associated with the programmes.

- Identify strategies used to implement the programmes within the trial.

- Identify potential links between implementation and clinical effectiveness of the programmes.

Such evaluation will also enable us to develop an evidence-based implementation blueprint to help guide the implementation, sustainment and scale-up of HARPdoc and/or BGAT after the trial is completed.

The study in this protocol depicts a complex research landscape. It is concerned with evaluating implementation of two educational programmes (ie, HARPdoc and BGAT) introduced and currently being tested within a parallel, two-arm, group randomised, blinded clinical trial, from the perspectives of multiple key intervention stakeholders (including, the adults with T1D, their relatives, as well as diabetes physicians, educators, psychologists and support staff). Within such a complex landscape, continuous stakeholder engagement is essential to the design of a meaningful implementation project. It is also critical in adequately addressing potential challenges and operational issues early on, thus helping maximise feasibility and relevance, in particular, in relation to the methodological approach and measures, as well as the subsequent data collection.

One such challenge was encountered in relation to the potential preintervention assessments. The complexity of assessing programme delivery within a parallel, two-arm, group randomised, blinded trial design means that the opportunity to assess course participants just before the start of the programme when they are fully aware of the arm that they are assigned to is small and dependant on the receipt of the ethical approval (for the implementation). This is further compounded by the methodological issues around surveying or interviewing programme participants about their views of the programmes while they are being randomly assigned to one or the other; something that could potentially contaminate the trial. Hence, the preintervention assessments in the current study were not feasible and the primary focus has been placed on postintervention measurements.
Nonetheless, the current study offers a stakeholderdriven mixed-methods approach to evaluating the implementation of two novel psychoeducational programmes in adults with T1D and problematic hypoglycaemia within the UK and US healthcare systems. While the difference in the two healthcare systems is fundamental, that is, public versus private provision of care, it provides an important insight into the breadth of potential barriers and facilitators to the implementation of such complex programmes, including any delivery adaptations potentially needed to achieve population coverage and scale-up; ultimately, enabling more people with T1D and problematic hypoglycaemia to benefit from the trialled programmes worldwide. Due to the complexity of the trialled interventions and the niche patient group that they are designed for, the inclusion of the specialist UK and US sites was pragmatically critical since they are leading tertiary referral centres with world renowned expertise in hypoglycaemia management, recognition and treatment.

The findings will, thus, inform the development of an implementation blueprint and the identification of specific implementation strategies for the post-trial scale-up of the programme/s into routine services. The findings will also form the basis for a further international trial (likely a hybrid type 3 effectiveness-implementation trial) ${ }^{35}$ focused on evaluating implementation success of a number of different implementation strategies in scaling up and sustaining the clinically effective intervention in different healthcare systems, following the current trial. Such developments will in turn contribute to the scientific understanding of methods for evaluating and implementing complex interventions within a complex organisational structure of a healthcare system, and within two different modes of care provision, that is, private/USA versus public/ UK, thus addressing the gaps on many important methodological and practical issues.

\section{ETHICS AND DISSEMINATION}

The study described in this protocol has been reviewed and given favourable opinion by the Research Ethics Committee (18/LO/1020; 240752) on October 01, 2018. Informed consent will be sought from all research participants for this study. The study will be conducted in accordance with the Good Clinical Practice and recommendations for physicians involved in research on human subjects adopted by the 18th World Medical Assembly, Helsinki 1964 and later revisions. The study will produce key information to the stakeholders on planning, funding and implementation of the interventions under investigation. Hence, the findings will be disseminated through peer-reviewed journals, relevant national and international meetings, as well as educational events within the individual hospitals to ensure that they are brought to the appropriate stakeholders. We will report to PWDs and their carers through publications of the Juvenile Diabetes Research Foundation, study website, and by the presentation to patient groups. 
Author affiliations

${ }^{1}$ Centre for Implementation Science, Health Service and Population Research Department, Institute of Psychiatry, Psychology and Neuroscience, King's College London, London, UK

${ }^{2}$ Department of Diabetes, School of Life Course Sciences, King's College London, London, UK

${ }^{3}$ Department of Diabetes, King's College Hospital, London, UK

${ }^{4}$ Department of Biostatistics and Health Informatics, Institute of Psychiatry, Psychology and Neuroscience, King's College London, London, London, UK ${ }^{5}$ Department of Diabetes, King's College London, People With Diabetes (PWD) Group: Mr Mike Kendall, Ms Lis Warren, Ms Victoria Ruszala, Ms Mel Stephenson, Mr Arthur Durrant, London, UK

\section{Twitter Tayana Soukup @TayanaSoukup}

Acknowledgements We would like to thank the representatives of the people with type 1 diabetes, as well as the health care professionals involved, for their time and commitment in the design of this study protocol. The collaborating authors for the PWD Group are Mr Mike Kendall, Ms Lis Warren, Ms Victoria Ruszala, Ms Mel Stephenson and Mr Arthur Durrant (London, UK).

Contributors As per International Committee of Medical Journal Editors guidelines, the coauthors have contributed as following: made substantial contributions to conception and design or acquisition of data, or analysis and interpretation of data; TS, LH, IB, AH, NS, SA, ELS and PWD group been involved in drafting the manuscript or revising it critically for important intellectual content. TS, LH, IB, AH, NS, SA, ELS and PWD group given final approval of the version to be published. Each author should have participated sufficiently in the work to take public responsibility for appropriate portions of the content; TS, LH, IB, AH, NS, SA, ELS and PWD Group agreed to be accountable for all aspects of the work in ensuring that questions related to the accuracy or integrity of any part of the work are appropriately investigated and resolved. TS, LH, IB, AH, NS, SA, ELS and PWD Group.

Funding The funding for this study was supported by the JDRF (4-SRA-2017-266M-N). LH, IB, SA and NS are funded by the National Institute for Health Research (NIHR) via the 'Collaboration for Leadership in Applied Health Research and Care South London' (CLAHRC South London) at King's College Hospital National Health Service (NHS) Foundation Trust, London, UK. LH and NS are also funded by King's Improvement Science, which is part of the NIHR CLAHRC South London and comprises a specialist team of improvement scientists and senior researchers based at King's College London. King's Improvement Science is funded by King's Health Partners (Guy's and St Thomas' NHS Foundation Trust, King's College Hospital NHS Foundation Trust, King's College London and South London and Maudsley NHS Foundation Trust), Guy's and St Thomas' Charity, the Maudsley Charity and the Health Foundation. NS is also funded by the South London and Maudsley NHS Foundation Trust. IB is supported by the NIHR Biomedical Research Centre at South London and Maudsley NHS Foundation Trust and by the NIHR Collaboration for Leadership in Applied Health Research and Care South London at King's College Hospital NHS Foundation Trust, King's College London. The funding agreements ensured the authors' independence in designing the study, interpreting the data, writing and publishing the report.

Disclaimer The views expressed are those of the authors and not necessarily those of the NHS, the NIHR or the Department of Health and Social Care.

Competing interests NS is the Director of London Safety \& Training Solution, which provides advisory and training services on safety and quality improvement to hospitals and training programs internationally. TS serves as a consultant to $F$. Hoffmann-La Roche Diagnostics providing advisory research services in relation to innovations for multidisciplinary tumor boards. The remaining authors declare that they have no competing interests.

Patient consent for publication Not required.

Ethics approval The study described in this protocol has been reviewed and given favourable opinion by the Harrow Research Ethics Committee (18/L0/1020; 240752) on 1 October 2018.

\section{Provenance and peer review Not commissioned; externally peer reviewed.}

Open access This is an open access article distributed in accordance with the Creative Commons Attribution Non Commercial (CC BY-NC 4.0) license, which permits others to distribute, remix, adapt, build upon this work non-commercially, and license their derivative works on different terms, provided the original work is properly cited, appropriate credit is given, any changes made indicated, and the use is non-commercial. See: http://creativecommons.org/licenses/by-nc/4.0/.
ORCID iD

Stephanie A Amiel http://orcid.org/0000-0003-2686-5531

\section{REFERENCES}

1 Seaquist ER, Anderson J, Childs B, et al. Hypoglycemia and diabetes: a report of a Workgroup of the American diabetes association and the endocrine Society. Diabetes Care 2013;36:1384-95.

2 Wild D, von Maltzahn R, Brohan E, et al. A critical review of the literature on fear of hypoglycemia in diabetes: implications for diabetes management and patient education. Patient Educ Couns 2007;68:10-15.

3 Pedersen-Bjergaard U, Pramming S, Heller SR, et al. Severe hypoglycaemia in 1076 adult patients with type 1 diabetes: influence of risk markers and selection. Diabetes Metab Res Rev 2004;20:479-86.

4 UK Hypoglycaemia Study Group. Risk of hypoglycaemia in types 1 and 2 diabetes: effects of treatment modalities and their duration. Diabetologia 2007;50:1140-7.

5 Akram K, Pedersen-Bjergaard U, Carstensen B, et al. Frequency and risk factors of severe hypoglycaemia in insulin-treated type 2 diabetes: a cross-sectional survey. Diabetic Med 2006;23:750-6.

6 Lawton J, Rankin D, Elliott J, et al. Experiences, views, and support needs of family members of people with hypoglycemia unawareness: interview study: table 1. Diabetes Care 2014;37:109-15.

7 Pedersen-Bjergaard U, Færch L, Allingbjerg M-L, et al. The Influence of New European Union Driver's License Legislation on Reporting of Severe Hypoglycemia by Patients With Type 1 Diabetes. Diabetes Care 2015;38:29-33.

8 Jacobson AM, Musen G, Ryan CM, et al. Long-Term effect of diabetes and its treatment on cognitive function. $N$ Engl J Med 2007;356:1842-52.

9 Patterson CC, Dahlquist G, Harjutsalo V, et al. Early mortality in EURODIAB population-based cohorts of type 1 diabetes diagnosed in childhood since 1989. Diabetologia 2007;50:2439-42.

10 Feltbower RG, Bodansky HJ, Patterson CC, et al. Acute complications and drug misuse are important causes of death for children and young adults with type 1 diabetes: results from the Yorkshire register of diabetes in children and young adults. Diabetes Care 2008;31:922-6.

11 Skrivarhaug T, Bangstad H-J, Stene LC, et al. Long-Term mortality in a nationwide cohort of childhood-onset type 1 diabetic patients in Norway. Diabetologia 2006;49:298-305.

12 Huxley RR, Peters SAE, Mishra GD, et al. Risk of all-cause mortality and vascular events in women versus men with type 1 diabetes: a systematic review and meta-analysis. The Lancet Diabetes \& Endocrinology 2015;3:198-206.

13 The healthcare quality improvement partnership NDA: national diabetes audit 2012-2013 report 2: Compl Mort 2015.

14 Centre THaSCl: the health and social care information centre, Hospital episode statistics for England. Inpatient statistics 2014:2012-3.

15 Geddes J, Schopman JE, Zammitt NN, et al. Prevalence of impaired awareness of hypoglycaemia in adults with type 1 diabetes. Diabetic Med 2008;25:501-4.

16 Hopkins D, Lawrence I, Mansell P, et al. Improved biomedical and psychological outcomes 1 year after structured education in flexible insulin therapy for people with type 1 diabetes: the U.K. DAFNE experience. Diabetes Care 2012;35:1638-42.

17 Rogers HA, de Zoysa N, Amiel SA. Patient experience of hypoglycaemia unawareness in Type 1 diabetes: are patients appropriately concerned? Diabet Med 2012;29:321-7.

18 Anderbro T, Gonder-Frederick L, Bolinder J, et al. Fear of hypoglycemia: relationship to hypoglycemic risk and psychological factors. Acta Diabetol 2015;52:581-9.

19 Rankin D, Elliott J, Heller S, et al. Experiences of hypoglycaemia unawareness amongst people with type 1 diabetes: a qualitative investigation. Chronic IIIn 2014;10:180-91.

20 Yeoh E, Choudhary P, Nwokolo M, et al. Interventions that restore awareness of hypoglycemia in adults with type 1 diabetes: a systematic review and meta-analysis. Diabetes Care 2015;38:1592-609.

21 Mühlhauser I, Jörgens V, Berger M, et al. Bicentric evaluation of a teaching and treatment programme for type 1 (insulin-dependent) diabetic patients: improvement of metabolic control and other measures of diabetes care for up to 22 months. Diabetologia 1983;25:470-6.

22 BERTIE type 1 diabetes education programme. Available: https:// www.bertieonline.org.uk/ [Accessed 12 September 2018]. 
23 Heller S, Lawton J, Amiel S, et al. Improving management of type 1 diabetes in the UK: the dose adjustment for normal eating (DAFNE) programme as a research test-bed. A mixed-method analysis of the barriers to and facilitators of successful diabetes self-management, a health economic analysis, a cluster randomised controlled trial of different models of delivery of an educational intervention and the potential of insulin pumps and additional educator input to improve outcomes. Programme Grants Appl Res 2014;2:1-188.

24 Elliott J, Jacques RM, Kruger J, et al. Substantial reductions in the number of diabetic ketoacidosis and severe hypoglycaemia episodes requiring emergency treatment lead to reduced costs after structured education in adults with type 1 diabetes. Diabet Med 2014;31:847-53.

25 National Institute for Health and Care Excellence. Type 1 diabetes in adults: diagnosis and management. PG. 17 recommendation 1.3.7. Available: https://www.nice.org.uk/guidance/ng17/resources/type1-diabetes-in-adults-diagnosis-and- management-1837276469701 [Accessed 12 Sep 2018].

26 Broers S, van Vliet KP, le Cessie S, et al. Blood glucose awareness training in Dutch type 1 diabetes patients: one-year follow-up. Neth $J$ Med 2005;63:164-9.

27 Kinsley BT, Weinger K, Bajaj M, et al. Blood glucose awareness training and epinephrine responses to hypoglycemia during intensive treatment in type 1 diabetes. Diabetes Care 1999;22:1022-8.

28 Schachinger $\mathrm{H}$, Hegar K, Hermanns N, et al. Randomized controlled clinical trial of blood glucose awareness training (BGAT III) in Switzerland and Germany. J Behav Med 2005;28:587-94.

29 Cox DJ, Gonder-Frederick L, Polonsky W, et al. Blood glucose awareness training (BGAT-2): long-term benefits. Diabetes Care 2001;24:637-42.

30 Cox D, Gonder-Frederick L, Polonsky W, et al. A multicenter evaluation of blood glucose awareness training-II. Diabetes Care 1995;18:523-8.

31 Hermanns N, Kulzer B, Ehrmann D, et al. The effect of a diabetes education programme (PRIMAS) for people with type 1 diabetes: results of a randomized trial. Diabetes Res Clin Pract 2013;102:149-57.

32 de Zoysa N, Rogers H, Stadler M, et al. A psychoeducational program to restore hypoglycemia awareness: the DAFNE-HART pilot study. Diabetes Care 2014;37:863-6.

33 Beyond education: a hypoglycaemia awareness restoration programme for people with type 1 diabetes and problematic hypoglycaemia persisting despite optimised care. Available: https:// www.harpdoc.org/ [Accessed 12 Sep 2018]

34 Amiel S, Choudhary $\mathrm{P}$, Jacob $\mathrm{P}$, et al. A group randomised controlled trial of a novel intervention addressing cognitions in adults with treatment-resistant problematic hypoglycaemia complicating type 1 diabetes therapy - the hypoglycaemia awareness restoration programme for people with type 1 diabetes and problematic hypoglycaemia persisting despite optimised self-care (HARPdoc). BMJ Open 2019;9:e030356.

35 Curran GM, Bauer M, Mittman B, et al. Effectiveness-implementation hybrid designs: combining elements of clinical effectiveness and implementation research to enhance public health impact. Med Care 2012:50:217-26.

36 The Medical Research Council. Developing and evaluating complex interventions: new guidance. Available: https://www.mrc.ac.uk/ documents/pdf/complex-interventions-guidance/

37 Craig P, Dieppe P, Macintyre S. Developing and evaluating complex interventions: new guidance: MRC 2008;337.

38 Craig P, Dieppe P, Macintyre S, et al. Developing and evaluating complex interventions: the new medical Research Council guidance. Int J Nurs Stud 2013;50:587-92.

39 Moore GF, Audrey S, Barker M, et al. Process evaluation of complex interventions: medical Research Council guidance. BMJ 2015;350.

40 Harden SM, Smith ML, Ory MG, et al. Re-aim in clinical, community, and corporate settings: perspectives, strategies, and recommendations to enhance public health impact. Front. Public Health 2018;6.

41 Proctor E, Silmere H, Raghavan R, et al. Outcomes for implementation research: conceptual distinctions, measurement challenges, and research agenda. Adm Policy Ment Health 2011;38:65-76.

42 Kirk MA, Kelley C, Yankey N, et al. A systematic review of the use of the consolidated framework for implementation research. Implementation Sci 2015;11.

43 Damschroder LJ, Hagedorn HJ. A guiding framework and approach for implementation research in substance use disorders treatment. Psychology of Addictive Behaviors 2011;25:194-205.

44 Powell BJ, Waltz TJ, Chinman MJ, et al. A refined compilation of implementation strategies: results from the expert recommendations for implementing change (ERIC) project. Implementation Sci $2015 ; 10$.

45 Implementation science research development (ImpRes) tool and guide. Available: http://www.kingsimprovementscience.org/files/ ImpRes_Guide_May2018_2.pdf [Accessed 3 Aug 2018].

46 Sweetnam C, Goulding L. Hull L. P14 Implementation Science Research Development (ImpRes) Tool Protocol Assessment Criteria (ImpResPAC): Development and Evaluation. Proceedings from the 2nd Annual UK Implementation Science Research Conference, 'Advancing the science of scaling up: Improving efficiency and effectiveness of implementation strategies in healthcare'. $/ \mathrm{mp} / \mathrm{Sci}$ $2019 ; 14$.

47 Weiner BJ, Lewis CC, Stanick C, et al. Psychometric assessment of three newly developed implementation outcome measures. Implementation Sci 2017;12.

48 Christopher CW, Ingersoll KS. Development and initial validation of the assessment of motivational interviewing groups - observer scales (AMIGOS). Int J Group Psychoth 2017;68:69-79.

49 Rixon L, Baron J, McGale N, et al. Methods used to address fidelity of receipt in health intervention research: a citation analysis and systematic review. BMC Health Serv Res 2016;16:663.

50 Fereday J, Muir-Cochrane E. Demonstrating rigor using thematic analysis: a hybrid approach of inductive and deductive coding and theme development. Int J Qual Methods 2006;5:80-92. 